\title{
Perioperative Management and Outcome after Surgical Treatment of Anterior Cerebral Artery Aneurysms
}

\author{
Issam A. Awad and John R. Little
}

\begin{abstract}
The authors present clinical experience with 28 cases of ruptured anterior cerebral artery (ACA) aneurysms managed personally during a 36 month period, and 10 unruptured ACA aneurysms. The cases included five giant aneurysms and four distal ACA aneurysms. Management strategy was uniform and included early operative intervention (except in the setting of deteriorating neurologic deficit, not attributable to hydrocephalus or hematoma), and vasospasm prophylaxis including calcium channel blockers and hypervolemic hemodilution and arterial hypertension. Modern diagnostic adjuncts including transcranial doppler were used as they became available. Good outcome (outcome grade l or 2) was observed at 6 months in $71 \%(20 / 28)$ of ruptured cases and in $90 \%$ (9/10) of unruptured cases; fair outcome (outcome grade 3 ) was observed in $14 \%(4 / 28)$ of ruptured cases and in $10 \%$ of unruptured cases; bad outcome (outcome grade 4 or 5 ) was observed in $14 \%(4 / 28)$ of ruptured cases. There were no instances of rebleeding after admission to the hospital. There was a single mortality in a patient moribund on admission. Delayed ischemic deterioration (DID) was documented in $46 \%$ (13 of 28) of the ruptured cases, and was a major source of morbidity in 7 of the 9 instances of fair or poor outcome in the series. Management outcome, including the occurrence of subtle neuropsychological difficulties commonly described in cases with ACA aneurysms, is discussed with relation to the incidence of DID, the clinical course of DID, and the possible impact of various therapeutic strategies.
\end{abstract}

RÉSUMÉ: Conduite péri-opératoire et issue du traitement chirurgical des anévrysmes de l'artère cérébrale antérieure. Les auteurs présentent leur expérience clinique de 28 cas d'anévrysmes rupturés de l'artére cérébrale antérieure (ACA) qu'ils ont traités personnellement sur une période de 36 mois et de 10 anévrysmes de l'ACA non rupturés. Les cas incluaient cinq anévrysmes géants et quatre anévrysmes distaux de l'ACA. La conduite du traitement a été uniforme et incluait une intervention chirurgicale précoce (sauf dans le contexte d'un déficit neurologique progressif, non attribuable à une hydrocéphalie ou à un hématome), et une prophylaxie du vasospasme, incluant des inhibiteurs des canaux calciques, de l'hémodilution hypervolémique et de l'hypertension artérielle. Des épreuves diagnostiques modernes ont été utilisées, incluant le doppler transcrânien, à mesure qu'elles devenaient disponibles. Une issue favorable (de grade 1 ou 2) a été observée à 6 mois chez 71\% (20/28) des cas avec rupture de l'anéverysme et chez $90 \%(9 / 10)$ des cas sans rupture; une issue passable (de grade 3 ) a été observée chez 14\% (4/28) des cas avec rupture et chez 10\% des cas sans rupture; une issue défavorable (de grade 4 ou 5) a été observée 14\% (4/28) des cas avec rupture. II n'y a eu aucun cas d'hémorragie récidivante après l'admission à l'hôpital. Il n'y a eu qu'un cas de décès chez un patient qui était moribond à l'admission. Une détérioration ischémique tardive (DIT) a été observée chez 46\% (13/28) des cas de rupture, et a constitué une source majeure de morbidité chez 7 des 9 cas dont l'issue était passable ou défavorable dans cette série. Nous discutons de la conduite du traitement des séquelles, incluant l'apparition de troubles neuropsychologiques subtils qu'on a souvent décrits chez les cas d'anévrysmes de l'ACA, en faisant état de l'incidence de la DIT, de l'évolution chinique de la DIT et de l'impact possible de différentes stratégies thérapeutiques.

Can. J. Neurol. Sci. 1991; 18: 120-125

Several controversies continue to surround the management of cerebral aneurysm. 1.2 Despite a clearly dilineated natural history, ${ }^{3}$ there is no uniform agreement about surgical indications, timing, or adjunctive therapeutic measures. In recent years, the advent of microsurgical techniques and meticulous intensive care management have impacted favorably on the outcome of patients with this disease. ${ }^{4}$
Morbidity from the initial hemorrhage, rebleeding following hemorrhage, and delayed ischemic deterioration (DID) from vasospasm remain major hurdles to favorable outcome. ${ }^{3,5}$ Only prophylactic surgery on unruptured aneurysm can prevent morbidity and mortality associated with subarachnoid bleeding itself. Once a subarachnoid hemorrhage has occurred, prompt diagnosis and intervention can be life saving. ${ }^{6}$ More recently,

From the Cerebrovascular Surgery Program, Department of Neurological Surgery, The Cleveland Clinic Foundation, Cleveland, Ohio Received September 26, 1990. Accepted November 26, 1990

Reprint requests to: Issam A. Awad, M.D., M.S., Department of Neurological Surgery, Desk S80, The Cleveland Clinic Foundation, 9500 Euclid Avenue, Cleveland, Ohio 44195 U.S.A. 
there has been renewed interest in early surgical intervention so as to decrease rebleeding and to allow more aggressive medical management of vasospasm and DID. ${ }^{7}$ It has also been suggested that early surgery might prevent vasospasm in some patients by clearing spasmogenic substances from the subarachnoid space. 7.8 .9 However, there continues to be concern that early surgical intervention might be more risky and could aggravate or worsen outcome from vasospasm.

These issues are particularly significant in midline aneurysms of the anterior communicating artery complex and other aneurysms of the anterior cerebral arteries (ACA). Vasospasm from such aneurysms often affects local perforating vasculature supplying vital septal regions and anterior hypothalamus. With such aneurysms often adjacent to the thin lamina terminalis of the third ventricle, it has been suggested that aneurysms in this location are particularly prone to rebleeding. Lastly, access for clipping of such aneurysms often requires brain retraction to substantial depth. This has raised concern about the advisability of early surgery for such aneurysms. Because of these concerns, management outcome from ACA aneurysms might be substantially different from that of aneurysms in other locations. Yet, little has been published about management outcome of ACA aneurysms specifically in the modern era of aggressive neurological intensive care and acute microsurgical intervention. Large multi-institutional series including several recently completed Cooperative Studies contain substantial numbers of patients with ACA aneurysms. In addition to the confusing factors of multiple surgeons, and multiple institutions, it is often difficult to sort out the particular outcome of patients with ACA aneurysms in such studies.

In this report, we present clinical experience with 38 consecutive cases of ACA aneurysms managed personally by one of the two authors. Management strategy was uniform, and consisted of early surgical intervention and aggressive compulsory management in a modern neurosurgical intensive care unit. Results are discussed with special attention to perioperative management strategies including the use of modern diagnostic adjuncts.

\section{Patients and Methods}

From June 1986 through May 1989, the authors personally managed 38 cases of ACA aneurysms. Ten cases underwent surgical intervention without evidence of previous hemorrhage (unruptured cases). Twenty-eight cases suffered subarachnoid hemorrhage, and were admitted to the hospital in various neurologic grades. The cases included 5 giant aneurysms and 4 distal ACA aneurysms. The age of the patients ranged from 26 to 72 (median age 47).

\section{Diagnosis and Preoperative Care}

In unruptured cases, the aneurysm was discovered incidentally on neuro-imaging studies performed for a variety of indications ( 8 cases), and in the process of evaluation of visual complaints ( 2 cases of giant aneurysms). In ruptured cases, the diagnosis of subarachnoid hemorrhage was usually suspected clinically, and was confirmed by computed tomography (CT) or lumbar puncture in every case. All patients had complete cerebral angiography via retrograde femoral artery catherization within 24 hours of admission. The aneurysm was demonstrated on initial angiogram in every case.
Upon establishing the diagnosis, hydration was initiated with crystalloids, and a central venous line was placed. A baseline evaluation with transcranial doppler (TCD) was obtained at the bedside in most cases. Patients were managed according to a uniform protocol (Figure 1).4,6 Early surgery (within 24 hours of admission) was performed except in the situation of deteriorating neurologic deficit not attributable to hematoma or hydrocephalus. The existence of angiographic spasm did not preclude early surgical intervention, nor did the clinical grade of the patient, unless there was severe symptomatic vasospasm or other untreatable cause of deteriorating neurologic status.

In the hours awaiting surgery (early cases) or in cases where surgery is necessarily delayed (as above), the patient is observed closely in the neurosurgical intensive care unit. In this early phase, cardiac arrhythmia and hyponatremia are prevalent. These are prevented and treated aggressively. Also, the patient is hydrated to restore intravascular volume (which is shown to be contracted in the few hours following subarachnoid hemorrhage). Hemodynamic objectives during this preoperative phase can best be described as normotensive hemodilution. In the presence of unclipped aneurysm, systolic blood pressure is not allowed to rise above 110 to $120 \mathrm{mmHg}$.

Precautions against rebleeding consist of controlling the systolic blood pressure (see above), prevention of vigorous cough, and prevention of excessive anxiety and straining. Barbituates and/or minor tranquilizers are extremely useful for this purpose.

Vasospasm prophylaxis is started early through hydration and the use of calcium channel blockers. Nifedipine was used early in the series, later to be substituted by Nimodipine when this was approved for clinical use in the United States. These drugs were administered in the normal recommended doses, but were withheld whenever the systolic blood pressure dropped below $90 \mathrm{mmHg}$.

Hydrocephalus was managed promptly by emergency ventricular drainage in all but good-grade stable patients. When ventricular drainage was instituted, intracranial hypotension was carefully avoided so as not to precipitate rebleeding. Drainage was typically used to maintain intracranial pressure in the range of $10-15 \mathrm{mmHg}$.

\section{Surgical Intervention}

Surgery was performed at the first availability of a fresh well-trained team of operating room nurses and technicians. This was typically the morning following admission to the hospital. During induction of anesthesia, close attention was paid to avoid wide swings in blood pressure. The head was fixed in 3 point head rest in a position of $30^{\circ}$ extension, and $30^{\circ}$ rotation to the side opposite that of the cranial opening. All cases were approached from the right side, except in the presence of hypoplastic right A-1 segment and an aneurysm dome projecting to the right. A C-shaped frontal temporal scalp incision was made extending just anterior to the hair line at the medial pupillary line. A free bone craniotomy was elevated flush with the floor of the frontal fossa. The cranial opening allowed a wide frontal pterional approach to the aneurysm. The pterional ridge was drilled to the superior orbital fissure to minimize brain retraction. The dura mater was opened parallel to the floor of the frontal cranial fossa and retractors were introduced under the frontal lobe to drain cerebral spinal fluid from the carotid cistern. This maneuver often allowed significant brain relaxation 


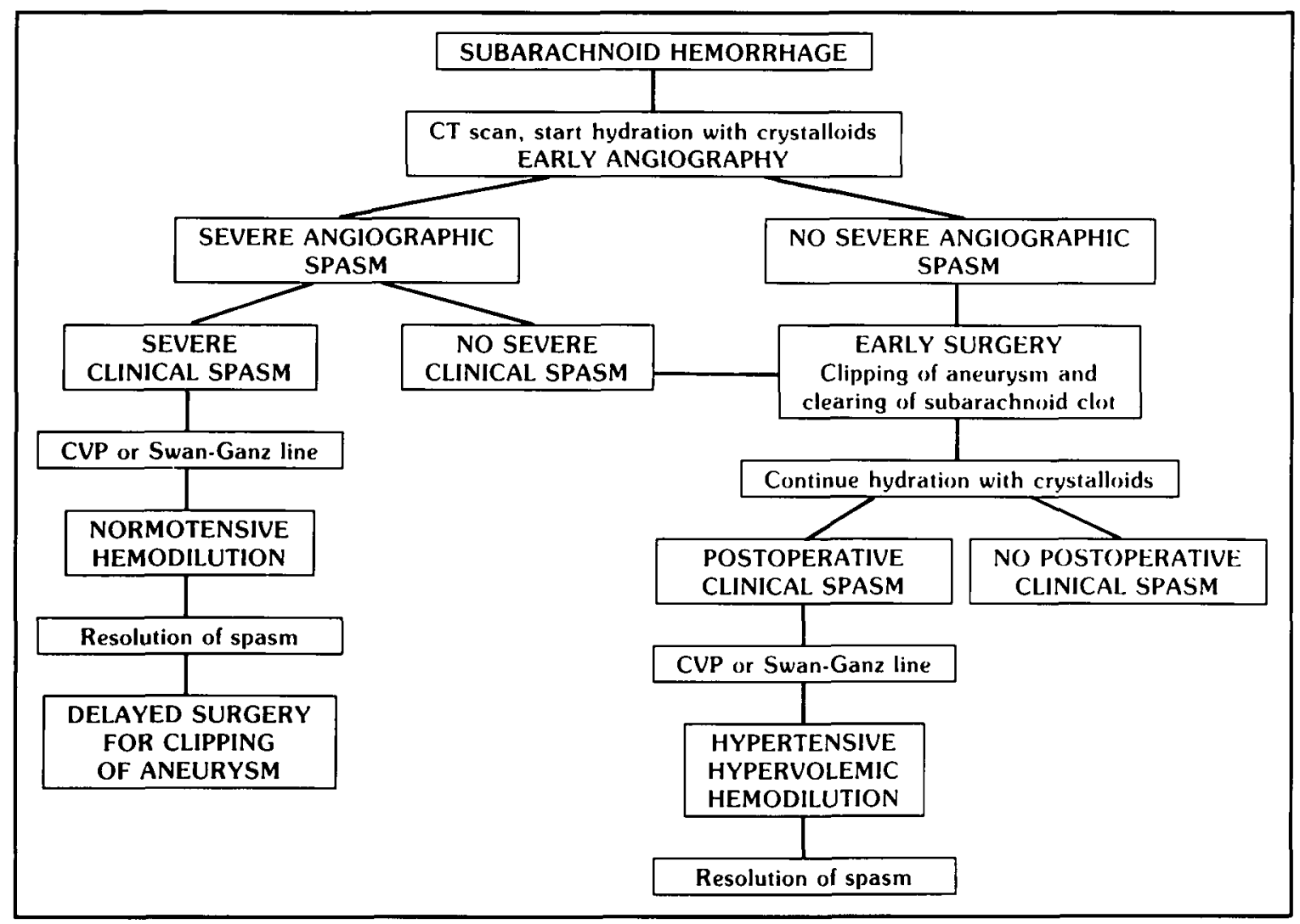

Figure I - Management Protocol for Subarachnoid Hemorrhage (adapted from Awad, et al.). ${ }^{6}$

despite the "red and angry" appearance of the brain hours after subarachnoid hemorrhage.

Hyperventilation and cerebral spinal fluid drainage allowed significant slackening of the frontal lobes so as to split the medial aspect of the sylvian fissure, and extend the arachnoidal opening medially over the optic chiasm. Systolic blood pressure was usually maintained around $100 \mathrm{mmHg}$ throughout the dissection. Induced hypotension was seldom used, except in rare instances of manipulation of the aneurysmal dome. The systolic blood pressure was increased slightly whenever temporary clips were applied.

After identifying the ipsilateral A-1 segment (and usually also the recurrent artery of Heubner), these were followed medially toward the anterior communicating artery complex. The gyrus rectus was coagulated and subpially suctioned to assist with the exposure of the anterior communicating artery complex. Both A-1 and A-2 segments of the anterior cerebral arteries were identified prior to any clip application. All visible perforating vessels were spared. Peculiar anatomic anomalies in the region were not uncommon, and were closely sought and studied by correlating the angiogram with intraoperative findings prior to final satisfaction with clip application.

In cases with distal ACA aneurysm, an interhemispheric exposure was performed with following of internal frontal branches in a centripetal fashion toward the branchpoint of the $\mathrm{ACA}$ where the aneurysm arose.

\section{Postoperative Management}

All patients were managed in the neurosurgical intensive care unit. Serial TCD studies were performed, typically every other day, to monitor for the development and course of vascular spasm. Clinical vasospasm was defined as delayed neurologic deterioration that could not be attributed to rebleeding, hydrocephalus, intracerebral hematoma, electrolyte abnormalities, or toxic or metabolic factors. $5,13,14$ Such delayed ischemic deterioration (DID) usually followed a stereotyped course, with gradual blunting of the level of consciousness starting 3-10 days after subarachnoid hemorrhage, followed within hours by focal neurologic deficits. At the first suggestion of clinical vasospasm, a Swan-Ganz pulmonary artery catheter was introduced over the existing central venous line. Simultaneously, the TCD was repeated and other necessary diagnostic tests were obtained to rule out other causes of delayed neurologic deterioration. Once the diagnosis of clinical vasospasm was established, hypervolemic hypertensive hemodilution was initiated.

The hemodynamic objectives of this treatment strategy consisted of raising the pulmonary wedge pressure to $15-18 \mathrm{mmHg}$, and maintaining the hematocrit in the $33-38 \%$ range. This was accomplished using colloid agents in volumes not exceeding $2,000 \mathrm{ml}$ per day. To accomplish the desired hematocrit levels, whole blood transfusion or phlebotomy was used as needed in anemic and polycythemic patients, respectively. In addition, the systolic arterial pressure was maintained in the $160-200 \mathrm{mmHg}$ range (except in the presence of unclipped aneurysm as noted above). Since many patients with subarachnoid hemorrhage have a spontaneous tendency toward arterial hypertension, the desired blood pressure objectives were usually accomplished passively with withdrawing some or all antihypertensive agents. Intravenous Dopamine (Intropin) drip or other pressors were used to assist with these objectives. 
Intensive monitoring and hypervolemic hypertensive hemodilution were maintained until resolution of clinical vasospasm and simultaneous return of TCD velocities to the normal range. At that time, postoperative angiography was repeated to confirm resolution of vasospasm, and also to document satisfactory clipping of the aneurysm.

\section{Grading Neurologic Status and Outcome}

For the purpose of this study, neurologic status was graded using a modified Hunt-Hess scale (Table 1). ${ }^{6}$ This was determined on admission in all patients, and was used to gauge progress during hospitalization, and also to determine outcome. All patients were followed for at least six months after surgery. Patients with suspected overt neurocognitive dysfunction (by subjective impression or by report from patient and especially from relatives) underwent detailed neuropsychological evaluation.

Table 1: Grading of Preoperative (Admission) State and Outcome After Subarachnoid Hemorrhage

\begin{tabular}{cll}
\hline \hline Grade & Preoperative (Admission) State & Outcome \\
\hline 1 & $\begin{array}{l}\text { No neurologic deficit; no } \\
\text { headache or mild headache }\end{array}$ & $\begin{array}{l}\text { Normal; resumed } \\
\text { occupation }\end{array}$ \\
2 & $\begin{array}{l}\text { Cranial nerve palsy; } \\
\text { severe headache; } \\
\text { meningismus; irritability }\end{array}$ & $\begin{array}{l}\text { Subtle difficulties; } \\
\text { does not require } \\
\text { assistance }\end{array}$ \\
3 & $\begin{array}{l}\text { Limb drift; drowsiness } \\
\text { (arousable by verbal stimulus) }\end{array}$ & $\begin{array}{l}\text { Moderate difficulties; } \\
\text { requires occasional } \\
\text { assistance }\end{array}$ \\
4 & $\begin{array}{l}\text { Limb paresis; aphasia; stupor } \\
\text { (arousable by painful stimulus) }\end{array}$ & $\begin{array}{l}\text { Requires major } \\
\text { assistance }\end{array}$ \\
5 & $\begin{array}{l}\text { Limb plegia or posturing; coma } \\
\text { (unarousable) }\end{array}$ & Vegetative or dead \\
\hline
\end{tabular}

\section{RESULTS}

\section{Timing of Surgery}

Despite a uniform management strategy aiming at surgical clipping as soon as possible after subarachnoid hemorrhage, early operative intervention could not be accomplished in every case. Table 2 lists the interval until surgery in relation to the timing of referral following subarachnoid hemorrhage. Only 8 of 28 $(28 \%)$ cases with subarachnoid hemorrhage reached our institution within 2 days of their bleed; all 8 cases underwent early surgery. Another 14 of the 28 patients $(50 \%)$ were referred at various intervals between the 3rd and the 15 th day after subarachnoid hemorrhage. Thirteen of these fourteen patients underwent surgical intervention without further delay (within 24 hours of their arrival to our hospital and correct diagnosis). One patient was referred on the 6th post-hemorrhage day in fulminant symptomatic vasospasm and deteriorating neurologic condition; surgery was delayed in this patient until the 15 th day. Five of the 28 cases (18\%) were referred after the 15th day following subarachnoid hemorrhage, and underwent surgery immediately upon arrival to our institution and confirmation of diagnosis.

\section{Incidence and Outcome of Delayed Ischemic Deterioration}

Overall, 13 of the 28 cases with subarachnoid hemorrhage (46\%) developed DID (as defined). The occurrence of DID in relation to the interval until surgery is listed in Table 2 . There was DID in 5 of $15(33 \%)$ cases operated in the first 7 days after subarachnoid hemorrhage; in all 5 cases, DID developed following surgical clipping of the aneurysm. There was DID in 8 of 13 $(61 \%)$ cases operated after the first week following subarachnoid hemorrhage; in all instances, DID was present prior to surgical clipping of the aneurysm. In some instances, the occurrence of DID allowed correct diagnosis of subarachnoid hemorrhage, and subsequent referral to our facility.

Table 3 lists the incidence of DID in relation to preoperative (admission) neurologic grade. The higher incidence of DID in cases with worse preoperative neurologic grade may be partly artifactual: some of these cases had a worse neurologic grade because of pre-existing DID (late referral cases).

All cases with DID were managed using calcium channel blockers and hypervolemic hypertensive hemodilution therapy. Despite late referral in many cases, 7 of the 13 patients with DID achieved a good final outcome (outcome grade 1 or 2), while 3 cases achieved a fair outcome (outcome grade 3 ), and 3 cases had a bad outcome (outcome grade 4 or 5 ).

\section{Overall Outcome}

Table 3 presents the overall outcome as assessed 6 months following subarachnoid hemorrhage. In general, the likelihood of good outcome decreased with worsened preoperative neurologic grade. A good outcome was observed at 6 months in $71 \%$ $(20 / 28)$ of ruptured cases and in $90 \%(9 / 10)$ of unruptured cases; fair outcome was observed in $14 \%(4 / 28)$ of ruptured cases and in $10 \%$ of unruptured cases; bad outcome was observed in $14 \%$ (4/28) of ruptured cases. The occurrence of DID was responsible for all but one instances of fair or bad outcome. In one case with preoperative grade 5 and acute akinetic mutism, the patient remained vegetative (outcome grade 5) without any definite evidence of DID.

It is important to note that $50 \%$ of patients grade 3 or worse

Table 2: Interval until Referral and the Timing of Surgical Intervention in 28 Cases of Ruptured Anterior Cerebral Artery Aneurysm

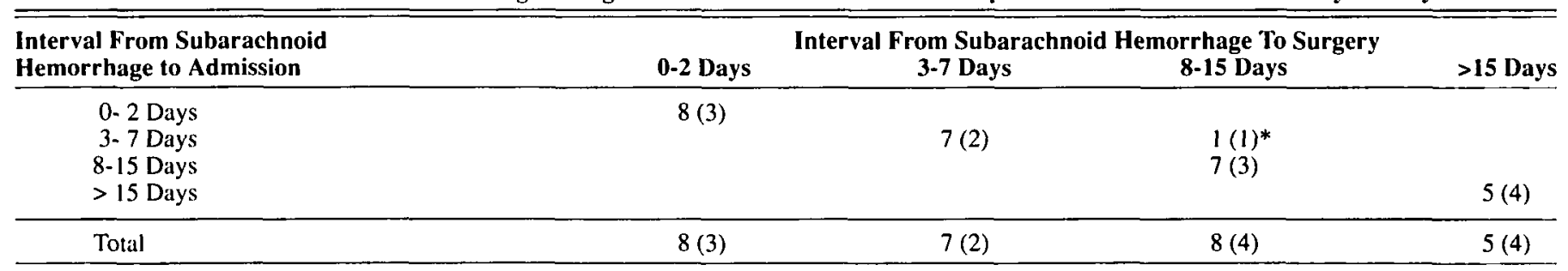

() Number of patients with delayed ischemic deterioration

* Surgery delayed because of progressive symtomatic vasospasm on arrival 
preoperatively eventually achieved a good outcome; half of the patients who were grade 5 preoperatively (moribund) achieved a fair postoperative outcome.

\section{Lateralization of Post-Operative Vasospasm}

There were 14 cases operated in the first week post-hemorrhage, and without preoperative angiographic, TCD, or clinical spasm. Of these cases, 6 developed postoperative angiographic and/or TCD spasm ( 5 cases had DID). All 6 cases had anterior communicating artery aneurysms.

In 5 of these 6 cases, postoperative spasm was clearly more severe contralateral to the craniotomy. In once case, spasm was equally severe bilaterally. There was not one instance where spasm was worse on the side of surgical approach.

\section{Mild Neuropsychological Dysfunction}

Of the 38 ruptured and unruptured cases in the series, 29 achieved a good outcome (outcome grade 1 or 2). Of these cases, there were 5 patients with persistent mild neuropsychological problems as identified subjectively by the patient, family, and/or confirmed by neuropsychological testing. There was not one typical clinical course or pathoetiologic mechanism which seemed to contribute to this outcome. Two of the cases followed uncomplicated surgery for unruptured aneurysm (no subarachnoid hemorrhage). One case was associated with each of the following scenarios respectively: grade 1 preoperatively without DID, grade 3 preoperatively with DID, and grade 4 preoperatively without DID.

\section{Discussion}

This report summarizes recent clinical experience at a modern tertiary referral center with consecutive cases of ACA aneurysm treated surgically. All cases were managed according to a uniform protocol geared toward acute clipping of the aneurysms. Compulsive management in the neurosurgical intensive care unit was followed according to a uniform protocol, and modern adjunct diagnostic and therapeutic measures were employed in every case.

Despite the uniform management strategy, treatment decisions were highly individualized. Surgery in the acute stage of subarachnoid hemorrhage was favored, so as to prevent rebleeding and to allow more aggressive management of vasospasm and DID. Yet, prior misdiagnosis and other referral factors prevented acute surgery in many instances. More often, early surgery at our center meant surgery "as soon as possible after admission" as opposed to "surgery within one or two days of subarachnoid hemorrhage". With this approach, there were no instances of rebleeding following admission to our institution.

Many patients presented to our service with angiographic and/or TCD evidence of spasm. This did not deter early surgical intervention, unless the patient was deteriorating neurologically from symptomatic spasm upon arrival (a single case). Other cases had already been through a phase of symptomatic spasm, and were in various phases of recovery with or without residual angiographic and/or TCD spasm

The incidence of postoperative spasm in this report was identical to that reported in various surgical series of subarachnoid hemorrhage. $2,6,10,11,13$ There is no evidence that early surgical intervention, even in the setting of existing spasm, precipitated or somewhat worsened ischemic sequelae. In fact, of 14 cases with mid-line aneurysm operated within the first week posthemorrhage and without preoperative angiographic or clinical spasm, there was not a single instance of angiographic and/or TCD spasm which is worse on the side of surgical approach and presumed vessel manipulation. This casts serious doubt on the hypothesis that surgical manipulation somehow precipates vasospasm. In fact, spasm was worse contralateral to the surgical approach in most instances, suggesting that the opening of basal cisterns on one side might have allowed clearing of spasmogenic substances.

Once DID was clinically manifested, the outcome was not uniformly bad. A highly individualized and aggressive therapeutic approach with all available modalities resulted in good outcome in the majority of these cases, and in fair outcome in a number of otherwise hopeless cases (grades 4 or 5 on admission to the hospital).

Overall management outcome was essentially related to the preoperative neurologic grade. However, an overall good outcome was accomplished in $90 \%$ of unruptured cases and in $75 \%$ of ruptured cases.

There has been serious concern about mild neurocognitive sequelae in patients with subarachnoid hemorrhage, especially cases with aneurysms in the ACA region. ${ }^{15}$ Using subjective and objective criteria, this was in fact a problem in $17 \%$ of our "good outcome" cases. Yet, this did not prevent most patients from resuming preoperative occupation and the majority of other societal functions. It is of interest that the pathoetiology of this complication was multifactorial. It occurred following surgery for unruptured aneurysms, and in cases with subarachnoid hemorrhage of various preoperative grades, with or without DID.

It is our conclusion that current surgical strategies have (nearly) eliminated rebleeding and surgical complications. Vasospasm remains the major factor in bad therapeutic outcome, although most cases with spasm in fact achieve a satisfactory

Table 3: Outcome Six Months after Surgery in Relation to Preoperative (Admission) Neurologic Grade

\begin{tabular}{|c|c|c|c|}
\hline Preoperative (Admission) Grade & Good (grade 1-2) & $\begin{array}{l}\text { Outcome Grade (6 Months) } \\
\text { Fair (grade 3) }\end{array}$ & Poor (grade 4-5) \\
\hline Unruptured & 9 & 1 & \\
\hline 1 & $9(2)$ & & \\
\hline 2 & $5(2)$ & $1(1)$ & $2(2)$ \\
\hline 3 & $4(1)$ & $1(1)$ & \\
\hline 4 & $2(1)$ & & \\
\hline 5 & & $2(2)$ & $2(1)$ \\
\hline
\end{tabular}

() Number of patients with delayed ischemic deterioration 
outcome. Subtle neuropsychiatric sequelae are common and are probably multifactorial. Despite a stated management strategy and an experienced team, early surgery remains often an elusive goal, in view of frequent early misdiagnoses and delayed referrals. Early surgical intervention upon admission to the hospital (regardless of time interval from hemorrhage) does not appear to prevent nor worsen spasm. Management strategies should be individualized within broad philosophic guidelines and specific therapeutic objectives. With this approach, the vast majority of patients with unruptured or ruptured ACA aneurysms derive significant benefit as compared to the unfavorable natural history of this disease, and eliminate the problem from further consideration during their lifetime.

\section{ACKNOWLEDGEMENTS}

This paper was presented at the Cerebrovascular Symposium on Anterior Cerebral Artery Aneurysms, hosted by the Faculty of Medicine of the University of Montreal and Notre-Dame Hospital on November 24,1989 . The Symposium was under the scientific direction of Professors Gerard Mohr and Yves Lamarre, and under the Honorary Presidency of Professor Guy Lazorthes.

\section{REFERENCES}

1. Ausman JI, Diaz FG, Malik GM, et al. Current management of cerebral aneurysms: is it based on facts or myths? Surg Neurol 1985; 24: 625-635.

2. Kassell NF, Boarini DJ, Adams HB, et al. Overall management of ruptured aneurysms: comparison of early and later operation. Neurosurgery 1981; 9: 120-128.

3. Sundt TM, Whisnant JP. Subarachnoid hemorrhage from intracra- nial aneurysms: surgical management and natural history of disease. N Engl J Med 1978; 299: 116-122.

4. Whiting DM, Barnett GH, Little JR. Management of subarchnoid hemorrhage in the critical care unit. Cleve Clin J Med 1989; 56: 775-785.

5. Kassell NF, Saskai T, Colohan AR, et al. Cerebral vasospasm following aneurysmal subarachnoid hemorrhage. Stroke 1985; 16: $562-572$

6. Awad IA, Carter LP, Spetzler RF, et al. Clinical vasospasm after subarachnoid hemorrhage: response to hypervolemic hemodilution and arterial hypertension. Stroke 1987; 18: 365-372.

7. Ljunggren B, Brandt L, Kagstrom E, et al. Results of early operations for ruptured aneurysms. J Neurosurg 1981; 54: 473-479.

8. Mizukami M, Kawase T, Usami T, et al. Prevention of vasospasm by early operation with removal subarchnoid blood. Neurosurgery 1982; 301-307.

9. Taneda M. Effect of early operation for ruptured aneurysms on prevention of delayed ischemic symtoms. J Neurosurg 1982; 57: 622-628.

10. Allen GS, Ahn HS, Preziosi TJ, et al. Cerebral arterial spasm: a controlled trial of Nimodipine in patients with subarachnoid hemorrhage. N Engl J Med 1983; 308: 619-624.

11. Kazner E, Sprung C, Adelt D, et al. Clinical experience with Nimodipine in the prophylaxis of neurological deficits after subarachnoid hemorrhage. Neuochirurgia 1985; 28: 110-113.

12. Espinosa $F$, Weir $B$, Overton $T$, et al. A randomized placebo-controlled-blind trial of Nimodipine after SAH in monkeys. Part I clinical and radiological findings. J Neurosurg 1984; 60: 1167 1175.

13. Chyatte D, Sundt TM. Cerebral vasospasm after subarachnoid hemorrhage. Mayo Clin Proc 1984; 59: 498-505.

14. Biller J, Godersky, JC, Adams HP. Management of aneurysmal subarachnoid hemorrhage. Curr Concepts Cerebrovasc Dis Stroke 1988; 23: 13-18.

15. Ropper AH, Zervas NT. Outcome one year after SAH from cerebral aneurysm. J Neurosurg 1984; 60: 909-915. 